\title{
Frontières
}

\section{Deuil post-traumatique sous l'empire de la terreur}

\section{Marie-Frédérique Bacqué}

Volume 15, numéro 2, printemps 2003

Guerre, mort amère

URI : https://id.erudit.org/iderudit/1073817ar

DOI : https://doi.org/10.7202/1073817ar

Aller au sommaire du numéro

Éditeur(s)

Université du Québec à Montréal

ISSN

1180-3479 (imprimé)

1916-0976 (numérique)

Découvrir la revue

Citer cet article

Bacqué, M.-F. (2003). Deuil post-traumatique sous l'empire de la terreur. Frontières, 15(2), 32-37. https://doi.org/10.7202/1073817ar

\section{Résumé de l'article}

Le nazisme a initié la systématisation de la terreur dans le cadre du génocide des Juifs : violences sur les civils, mutilations, désintégration des corps, disparition des restes. Les terroristes développent eux aussi la stratégie de la terreur totale, ajoutant aux victimes des attentats (blessés et morts), celles du traumatisme (les survivants et les endeuillés). Il ne s'agit donc plus seulement de tuer, mais aussi d'organiser l'horreur par la violence, l'aspect aléatoire de la mort, l'innocence des victimes. Cette hypothèse est vérifiée par le nombre de deuils post-traumatiques qui en découlent. L'absence de corps ou leur destruction empêchent une phase essentielle du travail de deuil : la prise de conscience de la réalité puis l'acceptation de la mort. Seule la vengeance peut émerger d'autant de douleur. Mais n'est-ce pas la menace du terrorisme : développer la spirale mortifère d’une guerre de position basée sur la rétorsion?
Ce document est protégé par la loi sur le droit d'auteur. L'utilisation des services d’Érudit (y compris la reproduction) est assujettie à sa politique d'utilisation que vous pouvez consulter en ligne.

https://apropos.erudit.org/fr/usagers/politique-dutilisation/ 


\section{Résumé}

Le nazisme a initié la systématisation de la terreur dans le cadre du génocide des Juifs: violences sur les civils, mutilations, désintégration des corps, disparition des restes. Les terroristes développent eux aussi la stratégie de la terreur totale, ajoutant aux victimes des attentats (blessés et morts), celles du traumatisme (les survivants et les endeuillés). II ne s'agit donc plus seulement de tuer, mais aussi d'organiser l'horreur par la violence, l'aspect aléatoire de la mort, l'innocence des victimes. Cette hypothèse est vérifiée par le nombre de deuils post-traumatiques qui en découlent. L'absence de corps ou leur destruction empêchent une phase essentielle du travail de deuil : la prise de conscience de la réalité puis l'acceptation de la mort. Seule la vengeance peut émerger d'autant de douleur. Mais n'est-ce pas la menace du terrorisme: développer la spirale mortifère d'une guerre de position basée sur la rétorsion?

Mots clés : terreur - deuil post-traumatiquetraumatisme - rites funéraires - horreur.

\begin{abstract}
Nazism has initiated systematization of terror in the Jewish genocide: violences on citizens, mutilations, disintegration of corpses, disparition of mortal remains. Terrorists develop total terror strategy too, adding to the victims of the attacks (wounded and deads), those of the traumatism (survivors and bereaved). They are not only killing people but they are organizing horror through violence, aleatory death and guiltlessness of victims. This hypothesis is verified by the importance of post-traumatic grieves. The lack of corpses or their destruction prevents essential stages of grief work : the awareness of reality then the acceptance of loss. Can only revange come into view of so much pain? Is'nt it the principal threat of terrorism : to develop the mortifying spiral of a position war based on retaliation?
\end{abstract}

Key words : terror-post-traumatic grief - traumatism - funerary rites - horror.

\section{DEUIL POST-TRAUMATIQUE SOUS L'EMPIRE DE LA TERREUR}

Marie-Frédérique Bacqué, Ph.D., vice-présidente de la Société française de thanatologie.

Assistons-nous à nouveau au phénomène de bascule de la domination entre l'Orient et l'Occident? Touchés dans leur ambivalence même (la suprématie économique sur les autres pays et l'angélisme devant la vitrine consumériste offerte aux pays les plus pauvres), les États-Unis et leurs alliés se voient maintenant combattus par une force obscure un grand réseau se réclamant, d'une justice « divine ». La lutte entre ces forces disparates peut pourtant être assimilée au combat d'un géant de fer face à un tigre de papier. Mais ce géant de fer et d'acier, terrassé par le petit moustique venu des airs (fixé dans nos esprits comme l'image de la tentative de destruction de l'omnipotence états-unienne à travers l'attaque de ses gratte-ciels), reste la métaphore à jamais inscrite dans nos mémoires du XXI ${ }^{\mathrm{e}}$ siècle, d'un possible renversement des choses. Paradoxalement, seul un combat souterrain pouvait aboutir à la visibilité du plus faible.

\section{LES CIVILS AU PREMIER PLAN DES VICTIMES DE GUERRE}

Depuis toujours, les actions «terroristes » constituent la façon la plus efficace «d'agacer» le système au pouvoir. Jadis, cependant, des personnalités politiques, de hauts représentants d'un État étaient assassinés par un individu ou un groupe. Aujourd'hui, c'est aux dépens de masses importantes de citoyens, souvent sans rapports directs avec la cause visée, que s'exerce le règne de la terreur. Ce phénomène s'inscrit dans une évolution historique de la guerre qui ne date pas de l'année 2001 (que nous pouvons dorénavant appeler «année du plus grand attentat commis contre des civils »), mais est lié à la disproportion des forces antagonistes : plutôt que de perdre la guerre contre des forces super armées, les islamistes de Ben Laden exercent une guérilla qui a bien plus d'impact par ses massacres de civils.

La guerre prend donc deux aspects : la forme académique, dans le déplacement spectaculaire et ordonné de masses de soldats vers un combat purement militaire, 
et la forme (mineure ?) de la guérilla larvée et du harcèlement chronique de la population civile d'un État ou d'une société par un groupe souterrain.

Dans les faits, et quelle que soit la modalité guerrière, les victimes civiles sont de plus en plus nombreuses alors que la guerre s'assimile à une mission pour des professionnels (Howard, 1986). Il y aurait donc, d'une part, des combats entre professionnels sur des actions ciblées et, d'autre part, des massacres de civils, dans le but de semer la terreur. Cependant, si ces ravages ont été observés lors de pogromes ou de génocides depuis quelques milliers d'années, la dernière guerre mondiale a inauguré de nouvelles souffrances. Ainsi, la mort « industrialisée » des Juifs par les nazis s'est avérée encore plus destructrice dans son « orientation mnémo-nécrologique » (Capdevila et Voldman, 2002, p. 159) : dès la guerre civile d'Espagne, en effet, les nazis interdirent que les Juifs qui y avaient trouvé la mort aient leurs noms gravés sur les monuments commémoratifs (Mosse, 1999, p. 201). Plus tard, l'annulation atomique d'Hiroshima et de Nagasaki confontera aussi les survivants à l'absence de restes de leurs proches.

Cette caractéristique d'anéantissement majore le traumatisme subi par une nation ou un groupe. Nous reviendrons sur cet interdit du souvenir des morts, dans le cas des attentats terroristes qui, volontairement ou involontairement, ne permettent pas la récupération des corps.

\section{IDENTIFICATION / \\ INDIVIDUALISATION ET PERTE DE SENS DES RITES FUNÉRAIRES}

Parmi les apports de Philippe Ariès, l'idée de l'évolution d'une mort publique vers une mort privée est une caractéristique de l'évolution des sociétés occidentales. Si la mort devient intime, c'est dans un contexte d'individuation des hommes et des femmes. Or, ce sentiment d'individualité si fort en Occident est littéralement contrebattu par le terrorisme « aveugle » qui frappe à n'importe quel moment, n'importe qui, sans prendre garde à l'identité des victimes.

De plus, aujourd'hui, un nouvel intermédiaire de poids a modifié la donne: il s'agit bien sûr des médias. Cet impact de la mort filmée ou photographiée de civils a été particulièrement bien compris par les terroristes. La télévision apporte la mort de "mon» voisin, de celui qui me ressemble, " chez moi ». Par le biais des identifications (également largement utilisées dans les divertissements de la "télé-réalité » où le quotidien est filmé jour et nuit; en France il s'agit d'émissions comme Le Loft), les terroristes, comme les nations en guerre, touchent le plus sûrement leur cible en montrant, par l'intermédiaire des médias, leur «double» aux téléspectateurs. Ainsi, lors des évacuations de populations du Kosovo, combien d'entre nous, Occidentaux, avons manqué défaillir en regardant ces colonnes de civils habillés en jeans, chaussant des Nike ou des anoraks de sportswear communs dans n'importe quel grand magasin occidental ? De même, lors de l'attentat du théâtre Doubrovka de Moscou en octobre 2002, aucune distance vestimentaire ne pouvait être opposée aux malheureux gazés, rendant du même coup les futurs spectacles beaucoup moins légers.

La télévision est le plus sûr moyen de faire entrer la terreur dans les maisons : demain ce peut être toi, «moi », qui seras touché. Cette mort au quotidien, cette mort «en boucle», est aussi la plus sûre façon d'accorder moins d'importance aux défunts et de les réduire à des acteurs du spectacle
Dans le cas des Palestiniens, les suicide bombers se camouflent pendant une période très courte, le temps de prendre le bus...

Pour les terroristes de septembre 2001, la feinte a été beaucoup plus longue, à la manière des espions dormants, qui intègrent vie professionnelle et familale dans le pays source de renseignements.

Donner la mort est cependant interdit dans toutes les civilisations et seule la guerre permet de transgresser ce tabou. Ce qui démontre bien que le réseau terroriste est en guerre contre les États-Unis et leurs alliés. Ce fait n'a, pendant longtemps, pas été clair par manque d'une communication explicite, mais aussi par le refus des États de se trouver en guerre contre une entité invisible, sans diplomatie, aux objectifs nébuleux. Nous verrons que l'absence de prise de conscience de l'état de menace par les citoyens des pays touchés est un facteur de traumatisme.
LE DEUIL NE CONCERNE PAS UNE PERSONNE MAIS UNE VICTIME PARMI D'AUTRES. IL EST DIFFICILE, DANS CES CONDITIONS, DE METTRE EN PLACE DES RITES FUNÉRAIRES CORRESPONDANT À LA VOLONTÉ ACTUELLE D'INDIVIDUALISATION COMMUNE EN OCCIDENT. audiovisuel de l'actualité. Ce regard permanent sur une mort «spectacularisée» (réduite en spectacle et non plus spectaculaire) déclenche une forme d'indifférence, voire de sidération face à la mort.

Or, la terreur résulte du «forçage » du mécanisme de défense collectif si bien mis en place en Occident depuis la fin de la Première Guerre mondiale (Gorer, 1995) : le déni de la mort et son corollaire, la perte de sens des rites funéraires. Le surgissement brutal de la mort à travers la « petite lucarne du quotidien » (la télé est d'ordinaire bien domestiquée) augmente le sentiment d'étrangeté dans un premier temps, puis de déni dans un second, avec ce phénomène de répétition qui avoisine le remâchage.

\section{LE TERRORISME SUR LA CORDE RAIDE DES IDENTIFICATIONS}

Nous ne pouvons pas assimiler les actions palestiniennes en Israël et celles du réseau al-Qaïda, cependant, retenons la similarité de la formation de «martyrs » qui, au sacrifice de leur vie, vont toucher l'Occident au plus profond, après s'être faussement intégrés.
Si l'Occident a perdu ses idéologies, si gagner de l'argent a remplacé la quête du salut de l'âme, la croyance religieuse semble soutenir une part du discours prétendant justifier les attentats. Elle fait, à notre avis, partie intégrante de la manipulation psychologique des terroristes, au même titre que le prétendu déicide de Jésus par les Juifs a servi longtemps de prétexte à l'antisémitisme.

Une autre particularité de la stratégie de manipulation des «combattants » consiste en un jeu d'identification-désidentification ordonné par leur chef. Les suicide bombers de Palestine sont tous très jeunes et issus de milieux très pauvres. Leur vie est économiquement difficile et socialement dévalorisée. Lorsqu'ils prétendent au suicide guerrier, c'est, pour eux, la seule façon de gagner en identité et véritablement de se subjectiver. C'est alors la mort et non la vie qui est valorisée par le groupe. Comme le souligne Christian Lachal (2002), les visages peints des martyrs figurent sur les murs des villages palestiniens, comme si seule leur mort les transformait enfin en personnes, idéalisées certes, mais ayant accès à une place durable. Ces martyrs peuvent être 
comparés aux suicidaires adolescents occidentaux: une expérience radicale permet de faire éclater leur avenir bloqué et leur donne l'illusion de changer le monde.

Mais même si les jeunes Palestiniens sont manipulés par des chefs de guerre, leur participation semble relativement «spontanée » en comparaison de celle des terroristes du World Trade Center.

La ruse et l'excellence de la connaissance du système occidental semblent ici primer sur l'enthousiasme morbide. Ces hommes relèvent plus du terrorisme guerrier que du terrorisme maquisard comme on pourrait appeler les attentats palestiniens. Ce qui frappe, c'est la durée de la préparation de l'attentat et la nature même de la «trahison» qui conduit un homme à s'insérer dans un réseau social puis à se défaire subitement de ses liens pour réaliser son œuvre de mort. Cette trahison suprême est d'autant plus cruelle que, finalement, ces «terroristes » dormants ont côtoyé leurs compatriotes d'infortune pendant longtemps. Ils n'hésitent pas à se retourner contre eux après tant de confiance. Cette identification des terroristes aux citoyens du pays d'accueil ne peut qu'amener méfiance à l'égard des étrangers résidant dans le pays. Il s'agit là d'un des effets pervers de ce mode de terrorisme basé sur l'identification : il engendre xénophobie et racisme.

\section{DE QUEL TYPE \\ EST LA PRÉPARATION \\ PSYCHOLOGIQUE DES \\ RÉALISATEURS D'ATTENTATS ?}

Les auteurs d'attentats actuels ont tous une idéologie sous-tendue par une référence à la religion musulmane. On pourrait pourtant citer l'IRA en Irlande et l'ETA dans le Pays basque espagnol, cependant, ces deux formations, si elles s'attaquent à des biens et des personnalités politiques, n'ont pas pour enjeu l'agression mortelle de personnes non liées explicitement au pouvoir en place.

D'aucuns diront du réseau al-Qaïda, qu'il est composé d'islamistes, c'est-à-dire de prosélytes de l'islam. Précisons qu'au sein des islamistes, seule une frange d'extrémistes prépare les actions terroristes. L'islam, par ailleurs, forme peut-être une «couverture » idéologique pour masquer des intérêts plus prosaïques de puissance chez les chefs de ce groupement.

Nous avons lu avec attention quelques documents publiés au lendemain des attentats de Manhattan, dans le journal français Le Monde et recueillis sur les lieux mêmes ou dans des valises «oubliées» par les terroristes. L'authenticité de ces textes, censés donner aux auteurs des détournements d'avions les dernières recommandations avant le moment clé, doit être mise en doute de propagande. Cependant, même si c'était le cas, trois éléments sont flagrants : logique particulièrement détaillée dans le Coran et reprise dans ces textes par allusions.

- l'insistance sur l'auto-obnubilation de la pensée, au moyen de prières répétitives ou de phrases toutes faites. Il s'agit de l'utilisation coercitive de pensées compulsives, pratiquée par tous ceux qui veulent limiter leur liberté de pensée ou calmer des fantasmes insupportables (en psychopathologie, ce phénomène est retrouvé dans le cas bien connu de la névrose de contrainte, plus couramment appelée névrose obsessionnelle).

- «l'obsessionnalisation » de la vie du terroriste passant par la répétition de rites de lavages (ablutions) et d'autres gestuelles débarrassant l'intéressé de ses souillures symboliques et réelles (et de ses crimes).

Ces trois caractéristiques, si elles n'émanent pas d'Al Quaida, ont peut-être été fabriquées, à des fins de propagande, par des spécialistes (Occidentaux) de la formation des bourreaux. Pour Françoise et il s'agit vraisemblablement de documents

- l'importance de la promesse eschato-

Sironi (1999, p. 142) : «Les processus sousjacents aux techniques initiatiques mises en œuvre pour former un tortionnaire et pour torturer une personne dans un système politique donné sont analogues aux mécanismes mobilisés lors de divers rituels initiatiques dans les sociétés traditionnelles. Les rituels initiatiques sont en fait des déclencheurs de nouvelles appartenances. » La thèse de Sironi est de dire qu'on ne naît pas bourreau, mais qu'on le devient. Elle emploie ce terme de bourreau pour désigner ceux qui sont formés aux techniques de torture physique et psychologique.

Dans le cas du mouvement al-Qaïda, nous imaginons deux niveaux: d'un côté, les acteurs des attentats qui ont été manipulés psychologiquement pour tuer un grand nombre de personnes, de l'autre, leurs « formateurs », véritables tortionnaires, qui ont su exploiter les difficultés psychologiques (voire psychiatriques), mais surtout, le manque d'insertion sociale de ces jeunes hommes et les orienter petit à petit vers le martyre guerrier.

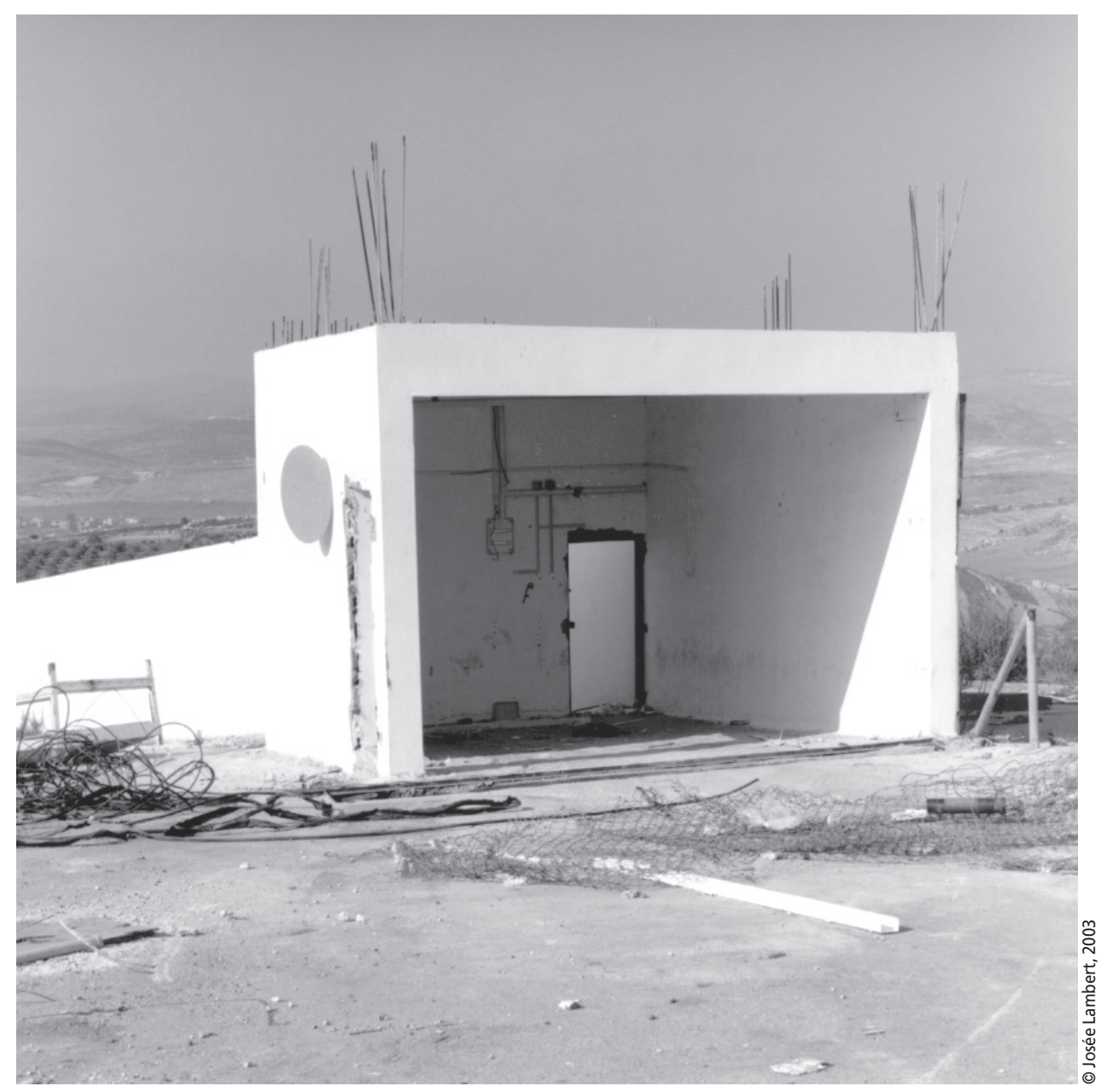

C'est visible, ces lieux servaient à vider de son humanité le vivant. 
Dans ce cas, même si l'on ne peut pas dire que les terroristes soient des bourreaux qui directement manipulent la souffrance de l'autre pour le faire avouer, il peut être envisageable de considérer qu'ils aient été formés sur ce mode pour, finalement, parvenir indirectement à l'assassinat de milliers de personnes. Parmi les mécanismes d'initiation à la pratique de tortionnaire, on retrouve des éléments proches de ce qui était écrit dans les documents appartenant aux terroristes :

- la séparation d'avec le monde social d'origine (transplantation aux États-Unis) ;

- la formation dans un milieu culturel différent (les camps d'Afghanistan) ;

- l'aspect initiatique de la formation qui ferait d'eux des hommes dignes de Dieu (mais dans la plupart des «formations» de bourreaux, il s'agit plus d'attribution de symboles de la virilité);

- à la perte des repères culturels initiaux succède l'accès à une nouvelle identité (le martyr) ;

- la stimulation de la haine de l'Autre, amalgamé en un "grand tout», hommes, femmes et enfants de la culture adverse honnie (chez les nazis qui faisaient partie des Sonderkommandos, on retrouvait la même pulsion de haine envers «le juif», entité globale déshumanisée).

C'est le système "désaffiliation-affiliation » à un nouveau groupe qui constitue le noyau de l'initiation, aussi bien dans la plupart des rites de passage de nombreux groupes humains que dans le cadre barbare de la formation de terroristes ou de bourreaux. Comme le remarque Sironi (1999, p. 137), « Les initiés accomplissent des travaux durs et pénibles dont la valeur repose sur la fierté, la beauté et l'excellence purifiée de leur nouvel état». Si nous ignorons la nature de la «formation» qu'ont subie les terroristes pendant leur préparation, la détermination avec laquelle ils ont accompli leur tâche mortifère (soit lorsqu'ils ont attaqué les pilotes, soit lors de l'assassinat de membres du personnel de bord) peut être mise en rapport avec la formation qu'avaient reçu d'anciens tortionnaires grecs (cité par Sironi, cet interview effectué par Peterson et Stephensen, date de 1982). Si notre hypothèse s'avère juste, on pourrait voir, dans la réalisation des attentats fomentés par al-Qaïda, une manipulation des terroristes, qui ne sont finalement que le bras armé d'un groupe beaucoup plus fort formé, lui, de tortionnaires, dont la vocation est de stimuler la haine et de donner des moyens d'action sous prétexte d'une idéologie hypocritement appliquée.
Le terroriste et le tortionnaire ne sont pas semblables : le tortionnaire est celui qui manipule psychologiquement le terroriste. Le terroriste n'interroge pas, n'a pas de contact humain avec sa victime, contrairement au bourreau qui va questionner sa victime et lui faire subir un traumatisme psychique. Dans la logique de la terreur, le tortionnaire fait subir le traumatisme de l'initiation au terroriste qui se transforme alors en une véritable machine de guerre.

\section{LES VICTIMES DE L'ATTENTAT}

Après avoir envisagé plusieurs hypothèses sur l'origine et la formation du terroriste actuel, nous allons évoquer les victimes de ces drames.

Comme précédemment, insistons sur le fait qu'il existe deux types de victimes du terrorisme : les personnes touchées directement par les armes employées et leurs proches. Notre article concerne les endeuillés, mais nous rendons hommage à toutes les victimes.

Nous nous appuierons sur notre expérience de psychothérapeute, travaillant essentiellement avec des patients en deuil d'un proche.

Toutes les conditions requises pour créer un traumatisme sont présentes dans les attentats du World Trade Center, de Bali puis de Moscou:

- l'effet de surprise et l'impuissance qui en découle;

- le contraste entre deux mondes: dans les tours jumelles, c'est le monde du travail (de la finance ?) utilisant les dernières technologies modernes et détachant l'homme des contingences matérielles primaires ou encore la fête et la détente dans le cas du restaurant-dancing de Bali, le spectacle pour Moscou;

- l'impréparation des victimes est aussi un facteur de traumatisme. Les militaires sont logiquement plus "préparés » à des attentats que des civils. Peut-on dire que les civils d'Israël ou d'Algérie sont plus préparés? Cela reste encore à prouver ;

- l'innocence des victimes, qui ne sont aucunement liées au conflit, mais qui ont en commun d'appartenir au groupe «ennemi », sans le savoir ;

- la brutalité et la violence de l'attentat qui se traduisent par une peur intense et un sentiment d'horreur pour les victimes directes et indirectes.

- l'amalgame entre tous les membres visés par l'attentat;

- la perte d'identité : les victimes n'existent plus en tant qu'individualités. Le traumatisme est encore plus puissant si elles ne peuvent être reconnues ou si leur corps a disparu;
- la médiatisation des événements qui donne une coloration mondiale au phénomène et déclenche un sentiment d'apocalypse.

\section{DÉFINITION D'UN NOUVEAU CONCEPT : LE DEUIL POST-TRAUMATIQUE}

Tous ces facteurs augmentent encore l'intensité de ce que nous appelons un "deuil post-traumatique » (Bacqué, 2002), c'est-à-dire le fait d'avoir perdu un proche, dans un contexte de violence, de brutalité et d'horreur. Ce concept de deuil posttraumatique se distingue du « deuil traumatique »(Prigerson et al., 1997, 1999), qui traite en fait de la souffrance traumatique liée au deuil, ce que nous appelons, pour notre part, un deuil traumatogène. En effet, dans le Traumatic Grief que décrit cette psychiatre se manifeste précisément l'incapacité à supporter la séparation d'avec l'être cher, quelle que soit la façon dont ce dernier a disparu. Cette incapacité se traduit par des complications du deuil, mais elle est liée à une inaptitude fondamentale à la séparation, inaptitude développée dès la petite enfance. Ici, les premières séparations (prise de conscience de la non-continuité du corps du bébé et de celui de sa mère, arrêt de l'allaitement, marche, croissance) ne fonctionnent pas comme des opérateurs du développement psychique, mais sont inconsciemment refusées. L'enfant, puis l'adulte éprouve un profonde nostalgie de cet attachement passé et, souvent, d'importantes difficultés à nouer de nouvelles relations et à se séparer des anciennes.

Pour nous, le deuil post-traumatique est spécifiquement composé des manifestations psychiques, comportementales et somatiques (ou de l'absence de signes) liées au vécu de la perte d'un proche dans un contexte traumatique.

Cette précision donnée, soulignons encore qu'il existe dans le deuil posttraumatique des facteurs exogènes à la personne, alors que dans le deuil traumatogène, les troubles et symptômes développés dépendent uniquement de la constitution de la personnalité, donc de facteurs endogènes.

\section{DEUIL POST-TRAUMATIQUE CONSÉCUTIF À UN ACTE DE VIOLENCE TERRORISTE}

Revenons aux situations de deuils posttraumatiques et insistons pour les différencier des états de stress post-traumatiques (DSM-IV), puisqu'il s'agit dans ce dernier cas de ce qu'éprouvent les victimes d'actes violents, alors que nous nous adressons aux endeuillés qui ont perdu un proche dans un contexte de violence et de brutalité. 
Tous les facteurs propres à l'attentat terroriste concourent à augmenter les risques de complications du deuil :

- la brutalité de la perte, sa violence et son caractère " anormal » dans le groupe social et culturel, ainsi que dans l'actualité historique du pays;

- la désorganisation de l'environnement familial et du groupe social (impact de l'événement sur la famille et tout le pays);

- le changement brusque de niveau économique de l'endeuillé en relation directe avec la disparition (perte de salaire du défunt mais surtout perte de son rôle social et familial);

- les difficultés de communication au sein du groupe (un excès de communication peut s'avérer aussi nocif que la carence de discours officiel. Il serait à cet égard important de comparer les populations de New York et de Moscou. Dans un cas, il y a eu information, dans l'autre carence quasi totale) ;

- l'absence de soutien au sein du groupe social élargi (déni de la mort ou de la violence, impossibilité d'évoquer les émotions liées au deuil ou au contraire «forçage » de la révélation des émotions alors que les endeuillés sont encore sidérés par l'événement).

Nous pourrions appliquer cet ensemble à la guerre, cependant la guerre est en général "plus établie» et la mort de soi et des autres peut atteindre un certain degré de prévisibilité. Dans l'attentat terroriste, l'effet de surprise est total et augmente la violence même de l'acte.

En revanche, un aspect partagé avec les catastrophes naturelles où le nombre des victimes est souvent très élevé et « où le hasard » est rendu responsable de nombreux décès concerne:

- La perte d'identité : l'impression de massification des victimes augmente cette idée de dépersonnalisation. Le deuil ne concerne pas une personne mais une victime parmi d'autres. Il est difficile, dans ces conditions, de mettre en place des rites funéraires correspondant à la volonté actuelle d'individualisation commune en Occident.

- L'aspect aléatoire de la mort: il apporte tous les jours sa part de regrets et de sentiments d'impuissance. «Pourquoi lui, elle? Pourquoi a-t-il pris l'avion, est-il retourné au bureau, est-il allé ce soir-là à la représentation?»

- La destruction du corps ou d'une partie du corps : les bombes, les mitraillages, les incendies ou les gaz rendent les corps méconnaissables et limitent les possibilités de reconnaissance des morts.

- La disparition des morts : pire encore est l'absence de cadavre, car elle bloque littéralement le travail de deuil. L'absence de corps empêche les phases principales des rites funéraires censés faciliter le travail de deuil. Les rites d'oblation et les rites de séparation (Thomas, 1985) ne peuvent être appliqués, puisqu'aucun corps ne peut être «soigné » (au sens de «to care » en anglais) dans un dernier effort pour le rendre digne et qu'aucun substitut de la personne ne pourra être symboliquement séparé des vivants.

L'absence d'objet métonymique du corps contribue à renforcer la difficulté de représentation de la mort de l'être cher et à prolonger le blocage du désinvestissement affectif (au sens où le travail de deuil implique un renoncement aux relations d'amour avec un être vivant mais une mise en histoire, une mise au passé de la relation d'amour).

\section{LIMITER LES DEUILS POST-TRAUMATIQUES}

Cette vaste question nécessite un article à elle seule. Nous allons articuler cependant notre pratique de deuils post-traumatiques à l'actualité des attentats terroristes. Insistons tout d'abord sur la longueur et la nocivité de ces deuils post-traumatiques non traités. Nous avons rencontré des personnes qui, quarante ans après les pertes familiales et les tortures infligées en camps de concentration, ressentaient toujours les affres du deuil post-traumatique (Bacqué, 1992). Dans le cas d'accidents de la route, certains endeuillés étaient à proximité de leur parent blessé à mort et avaient euxmêmes subi les conséquences du choc. Enfin, après une catastrophe naturelle (tremblement de terre en Arménie) ou humaine (accident du Concorde en France), nous avons rencontré des familles en état de choc et en difficulté pour mener leur deuil. Notre expérience est donc indirecte sur le plan du terrorisme, mais approchante quant aux conséquences sur les personnes rencontrées.

La sidération est toujours première dans les deuils post-traumatiques, mais si l'on ne peut jamais dire qu'un deuil soit attendu, on peut mettre sous ce critère un facteur de subjectivité majorant l'impact du traumatisme. La sidération se traduit par un affaiblissement des conditions vitales du sujet qui va de l'incapacité à penser jusqu'à l'impossibilité de se nourrir. Cet état de choc se traduit sur le plan physique par des difficultés respiratoires (dyspnée) dues vraisemblablement à un syndrome anxieux déclenché par la peur de la mort, par des difficultés digestives et par des troubles du sommeil (difficultés d'endormissement, cauchemars et reviviscences envahissant le sommeil). Sur le plan cognitif, les capacités de concentration et de remémoration sont touchées, une certaine obnubilation limite tout nouvel investissement intellectuel. Enfin, d'un point de vue psychologique, une impression d'anesthésie affective découle des efforts faits pour éviter les pensées et les souvenirs du traumatisme, mais aussi des tentatives de détachement des autres et de tout ce qui comptait affectivement auparavant. La réactivité du sujet est à la fois émoussée et suractivée (hypervigilance) dans les conditions rappelant le traumatisme. En effet, des réactions de sursaut, d'agissement automatique surviennent et gênent la personne, qui a l'impression de ne plus rien maîtriser.

Á ces aspects typiques du choc posttraumatique se surajoute le deuil, c'est-àdire le chagrin de la perte et la dépression du manque et de l'absence.

Le deuil post-traumatique doit donc être traité sur deux plans successifs: l'état de choc puis l'accès aux émotions négatives du chagrin.

Létat de choc va être abordé classiquement (dans une perspective psychanalytique, c'est-à-dire de reconnaissance de la place de l'inconscient et des relations tranféro-contre-transférentielles), sans «forçage », avec respect du silence de l'autre, éthique et dialogue.

Préciser sur quel bord on se situe et savoir que, d'un point de vue professionnel, on est aussi atteint par l'importance de la médiatisation et donc susceptible de ressentir soi-même une part du choc, fait partie de la réflexion éthique à tenir avant de proposer une psychothérapie. Aborder ses références professionnelles peut également être nécessaire si la personne est très méfiante après avoir subi une forme de trahison de sa confiance. Le travail psychothérapique consiste essentiellement à créer $\mathrm{du}$ lien. Lien historique entre l'avant et l'après, lien explicatif entre l'événement et ses conséquences, lien transférentiel entre le disparu et le thérapeute, lien entre les sentiments ambivalents ressentis à l'égard du défunt. Ce lien, avant de figurer dans le discours du patient, doit prendre corps dans ses représentations. Or les représentations de l'événement traumatique sont systématiquement chassées par le traumatisé. Il est donc nécessaire d'établir une relation suffisamment confiante pour qu'une réactualisation mnésique puisse avoir lieu sous forme de souvenir, plus que sous forme de reviviscence ou flash-back automatique, non maîtrisable. Le « film » de l'événement est vivid, comme disent les Anglais, c'està-dire, comme si l'on y était (il s'agit de 
l'enregistrement cérébral des données de l'événement, particulièrement réaliste, puisque l'hypervigilance était nécessaire pour utiliser toute possibilité de survie). Il doit progressivement être transformé pour passer de l'image à la représentation, puis de la représentation de choses à la représentation de mots (Freud, 1952, p. 155-156).

Ainsi, la possibilité de se représenter puis de symboliser les événements va permettre l'accès à la métabolisation psychique de l'événement et à son intériorisation. La mentalisation de nouveau accessible (Bacqué et Hanus, 2000) permettra l'accès à la dépression consécutive à la reconnaissance de la perte. Le chagrin est en effet le corollaire intime de la phase de séparation collective reconnue lors des rites funéraires.

Nous venons d'aborder le travail psychothérapique individuel avec une personne présentant un deuil post-traumatique. Il reste aux États victimes d'attentats terroristes sur leur territoire à préparer ce travail individuel par des cérémonies collectives.

Tout deuil se situe en effet au carrefour du social et du psychologique (Bacqué, 1997, p. 250). Dans les cas extrêmes de mort collective et violente, un groupe humain, une nation peut effectuer une part du travail de représentation et de symbolisation en montrant sa cohésion dans la réprobation du crime, mais aussi dans une cérémonie redonnant leur dignité aux victimes. Les cérémonies collectives permettent:

- une reconnaissance de la mort par la société ;

- une expression des émotions du deuil, bloquées par la sidération;

- elles redonnent une dignité aux morts et, par un phénomène de métonymie (un cercueil vide ou une silhouette symbolise le mort), permettent d'accéder aux rites d'oblation;

- enfin, la cérémonie collective ramène une forme d'ordre dans la société, qui vit la mort violente comme une augmentation extrême d'entropie sociale.

Ce retour de la dignité est, à notre avis, le seul acte susceptible d'agir sur les terroristes eux-mêmes. En effet, il ne s'agit plus pour la nation atteinte de déclencher la rétorsion, mais de montrer qu'elle oppose à la barbarie, l'humanité et à la déculturation de l'homme machine, la culture des rites sociaux. La vengeance n'est en effet qu'une surenchère de violence et conduit là où les attaques voulaient mener: une guerre de position stérile.

Il semble difficile de faire succéder à la violence, le dialogue. Mais parallèlement à la diplomatie, toutes les tentatives de communication doivent être faites. C'est seulement à ce prix que les attitudes désespérées du «terrorisme martyre » et perverties de la manipulation de bombes humaines diminueront peu à peu.

\section{Bibliographie}

ARIÈS, P. (1977). L'Homme devant la mort, Paris, Seuil.

BACQUÉ, M.-F. (2002). Deuil et catastrophes naturelles, Communication au $6^{\mathrm{e}}$ congrès de la Fédération européenne Vivre son deuil. Paris.

BACQUÉ, M.-F. et M. HANUS (2000). Le deuil, Paris, PUF, coll. «Que sais-je? ».
BACQUÉ, M.-F. (dir.) (1997). Mourir aujourd'hui. Les nouveaux rites funéraires, Paris, Odile Jacob.

BACQUÉ, M.-F. (1992). Le deuil à vivre. Paris, Odile Jacob. (1995) : Deuxième édition revue et augmentée. (2000): Troisième édition. Traductions en allemand et en grec.

CAPDEVILA, L. et D. VOLDMAN (2002). Nos morts. Les sociétés occidentales face aux tués de la guerre, Paris, Payot.

FREUD, S. (1952). "L'inconscient», dans Métapsychologie, Paris, Gallimard.

GORER, G. (1995). Ni pleurs ni couronnes, Paris, EPEL.

HOWARD, M. (1986). La Guerre dans l'histoire de l'Occident, Paris, Fayard.

LACHAL, C. (2002). Deuils et terrorisme. Approche endogène du terrorisme martyr, Communication au $6^{\mathrm{e}}$ congrès de la Fédération européenne Vivre son deuil. Paris.

MOSSE, G.W. (1999). De la Grande Guerre au totalitarisme. La brutalisation des sociétés européennes, Paris, Hachette.

PETERSON, J. F. et E. STEPHENSEN (1982). Le Fils de ton voisin, Centre de documentation vidéographique d'Amnesty International.

PRIGERSON, H., A.J. BIERHALS, S.V. KASL et al. (1997). "Traumatic grief as a risk factor for mental and physical morbidity ", American Journal of Psychiatry, vol. 154, $\mathrm{n}^{\circ} 5$, p. 616-624.

PRIGERSON, H., S.C. JACOBS et al. (1999). "Consensus criteria for traumatic grief», British Journal of Psychiatry, $\mathrm{n}^{\circ}$ 174, p. 67-73.

SIRONI, F. (1999). Bourreaux et victimes. Psychologie de la torture, Paris, Odile Jacob.

THOMAS, L.-V. (1985). Rites de mort. Pour la paix des vivants, Paris, Fayard. 